\title{
Évaluation de la durabilité_ INAA Prod.Anim, de la filière Indication Géographique Protégée « Canard à foie gras du Sud-Ouest »
}

Laura FARRANT', Marie-Pierre LABAU', Martine PADILLA², Chloé DENEUFBOURG ${ }^{3}$, Laurence FORTUN-LAMOTHE ${ }^{3}$, Sophie PENAVAYRE ${ }^{4}$, Antoine BESNIER ${ }^{5}$

${ }^{1}$ CTCPA, 2 allée Dominique Serres, 32000, Auch, France

${ }_{2}^{2}$ CIHEAM-IAMM, 3191 route de Mende, 34090, Montpellier, France

${ }^{3}$ GenPhySE, Université de Toulouse, INRA, INP-ENVT, 31320, Castanet-Tolosan, France

${ }^{4}$ IFV, 210 Bd Victor Vermorel, CS 60320, 69661, Villefranche-Sur-Saône, France

${ }_{5}^{5}$ ITERG, Parc Industriel Bersol 2, 11 Rue Gaspard Monge, 33600, Pessac, France

Courriel : Ifarrant@ctcpa.org

L'évolution des attentes sociétales entraîne de nouveaux questionnements pour les filières agroalimentaires et notamment les filières d'élevage : impact des productions animales sur le changement climatique, connaissance des circuits de production, transformation et distribution par les consommateurs, etc. Pour une filière sous Signe Officiel de Qualité telle que la filière sous Indication Géographique Protégée « Canard à foie gras du Sud-0uest », un préalable repose sur une meilleure connaissance des impacts environnementaux et sur l'évaluation de son poids économique et social pour son territoire.

\section{Introduction}

Le développement durable est devenu une préoccupation pour l'ensemble des parties prenantes des filières agro-alimentaires françaises : consommateurs, agriculteurs, distributeurs, pouvoirs publics. La prise en compte des enjeux associés aux dimensions multiples du développement durable nécessite d'intégrer les principes de la «pensée cycle de vie » au sein des filières alimentaires, en se préoccupant des impacts environnementaux, économiques et sociaux des pratiques agricoles, des modes de production, de distribution et de consommation. Aujourd'hui, l'analyse de cycle de vie (ACV) des produits est un outil largement utilisé pour évaluer les impacts environnementaux potentiels des activités de production.
En ce qui concerne les produits alimentaires transformés, quelques entreprises ont pris l'initiative de réaliser des ACV environnementales de leurs produits. De plus, des travaux méthodologiques ont été menés ces dernières années: Agribalyse (Koch et Salou, 2014) ; initiative européenne du « Product Environmental Footprint (PEF) Guide » (Commission Européenne, 2013) ; guides méthodologiques " Livestock Environmental Assessment and Performance (LEAP) » (FAO, 2016). Toutefois, la recherche de consensus méthodologiques ainsi que la constitution de bases de données exhaustives et représentatives sont toujours en cours et nécessaires.

Au-delà des considérations environnementales, en ce qui concerne les aspects économiques et sociaux, la conceptualisation et la mise en œuvre des méthodologies d'évaluation sont en discussion. Les scientifiques et les professionnels cherchent à intégrer des indicateurs de performances socio-économiques des systèmes productifs à travers des ACV sociales, des démarches prenant en compte la Responsabilité Sociétale des Entreprises (RSE) et d'autres indicateurs d'impacts territoriaux (tels que l'emploi et la richesse créés sur le territoire). Les outils adéquats restent ainsi encore rares et d'appropriation complexe.

Le projet « Durabilité environnementale, sociale et territoriale des produits alimentaires transformés » (ACYDU), financé par l'Agence Nationale de la Recherche (ANR), cherche à développer des méthodologies permettant d'évaluer la durabilité des filières agroalimentaires. Pour ce faire, le projet étudie les impacts environnementaux, sociaux, économiques 
et territoriaux des filières de production de trois produits français emblématiques sous signe de qualité : IGP foie gras du Sud-Ouest, vins AOP de Beaujolais et Bourgogne et AOP Comté. Ces filières ont été choisies car i) elles sont représentatives d'une culture et d'un savoir-faire traditionnel français; ii) leurs produits sont largement consommés sur le territoire ; iii) elles ont un lien fort avec la production agricole et $i v$ ) revendiquent un ancrage territorial important. Cependant, elles sont également pour diverses raisons questionnées par les parties prenantes sur certaines de leurs pratiques et doivent trouver des réponses.

Cet article présente les développements méthodologiques proposés et les résultats obtenus pour la filière IGP foie gras du Sud-Ouest. L'IGP canard à foie gras du Sud-Ouest couvre l'ensemble des bassins traditionnels d'élevage, de gavage et de transformation du foie gras : Chalosse, Gascogne, Gers, Landes, Périgord, Quercy. L'appellation garantit que les canards ont été élevés, abattus et transformés dans cette zone géographique ainsi que le respect d'un cahier des charges précisant notamment des conditions d'élevage et de gavage. Cette filière fait face à des enjeux de différentes natures. Ainsi, d'un point de vue sociétal la filière est confrontée à des questions telles que la prise en compte du bien-être animal, la gestion du travail saisonnier (nature des contrats de travail, organisation des entreprises pour le recrutement et l'encadrement de ces personnes et pour la planification de la production) ou encore la gestion de la crise sanitaire liée à I'Influenza aviaire. La filière est également concernée au même titre que les autres filières animales par la question de l'empreinte environnementale des procédés d'élevage, aujourd'hui au cœur de débats politiques et citoyens reflétés récemment au travers des États Généraux de l'Alimentation.

Le projet ACYDU se traduit en pratique par une collaboration interdisciplinaire entre des spécialistes des filières agro-alimentaires étudiées (instituts techniques agro-alimentaires, interprofessions, syndicats), des chercheurs en évaluation des impacts environnementaux, économiques et sociaux et des spécialistes de l'approche consommateur. Le projet propose ainsi de contribuer à la production de connaissances conceptuelles et empiriques sur les méthodologies d'analyse du cycle de vie qui intègrent les différents aspects de la durabilité des produits destinés à l'alimentation humaine. Sont aussi étudiés le potentiel de reproductibilité de ces méthodes à d'autres filières alimentaires et leur appropriation par les agro-industries pour l'évaluation de la durabilité des produits mis sur le marché.

\section{Bilan environnemental de la filière IGP foie gras du Sud-Ouest}

\subsection{Cadrage de l'étude}

L'objectif de ce travail d'évaluation environnementale est double: proposer d'une part un cadre méthodo- logique basé sur l'ACV permettant d'étudier non plus un produit ou une organisation mais l'ensemble d'une filière; et améliorer d'autre part la connaissance des impacts environnementaux de la filière étudiée. Dans le projet ACYDU, la notion de filière, représentée sur la figure 1, regroupe:

i) la « filière de production et de distribution » composée d'un cœur de filière incluant les entreprises et organisations directement impliquées dans la production du foie gras (éleveurs, gaveurs, abattoirs, ateliers de découpe, transformateurs/conditionneurs), auquel s'ajoutent les acteurs de l'amont (sélectionneurs, accouveurs, fournisseurs d'aliments...) et de l'aval (distributeurs, consommateurs...);

ii) I'« environnement proche » comprenant les structures « support» : organisme

Figure 1. Acteurs de la filière et principaux flux de matières et d'information.

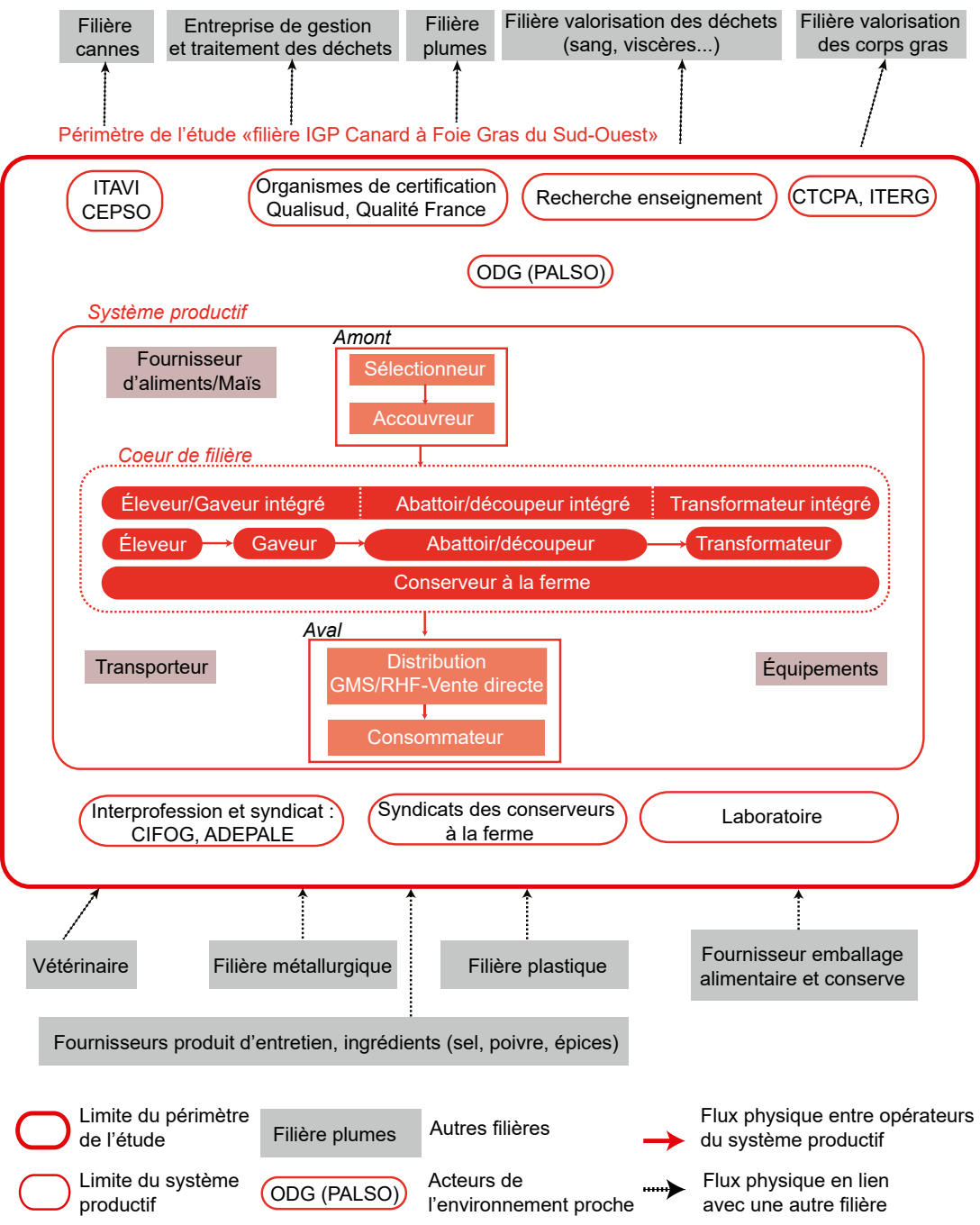


de défense et de gestion (ODG), syndicats, interprofessions, chambres d'agriculture, organismes de recherche et développement, organismes d'inspection et de certification.

La réunion de l'ensemble des activités de ces acteurs et structures compose le périmètre étudié appelé « filière IGP canard à foie gras du SudOuest ». La définition du périmètre de la filière résulte d'un travail méthodologique préliminaire réalisé dans le cadre des premières phases du projet ACYDU (Assogba, 2015). L'étape amont de production d'œufs et accouvage n'a néanmoins pas été incluse dans le périmètre de l'ACV, faute de données disponibles. Les frontières du système étudié commencent ainsi à partir de la « mise en place » des canetons mâles à un jour. Les étapes de traitement et de valorisation des coproduits (plumes, viscères...) ayant lieu après la phase de découpe ne sont pas attribuées à la filière foie gras et ne sont donc pas prises en compte dans le périmètre étudié.

Le choix de travailler à l'échelle d'une filière dite " environnée " a impliqué des développements méthodologiques spécifiques réalisés à partir du cadre général de l'ACV pour permettre la constitution de l'inventaire de cycle de vie. L'unité fonctionnelle (encadré 1) retenue est la quantité de produits finis commercialisés par la filière IGP foie gras du Sud-Ouest pour une année de

Encadré 1. L'unité fonctionnelle dans une analyse de cycle de vie.

L'ACV, Iorsqu'elle est appliquée à un produit, bien ou service, ou encore à une organisation, est encadrée par des normes internationales (ISO, 2006a, b; AFNOR, 2015) permettant de guider sa réalisation. La définition des objectifs et du champ de l'étude constitue ainsi la première étape. Il est notamment essentiel de préciser les frontières du système étudié et l'unité fonctionnelle retenue. L'unité fonctionnelle est I'unité de mesure à laquelle sont rapportés les impacts environnementaux au moment de l'expression des résultats. L'unité fonctionnelle retenue ici est la quantité de produits finis commercialisés par la filière IGP foie gras du Sud-Ouest pour l'année civile 2014.

Encadré 2. Méthodes de caractérisation des indicateurs d'impacts retenus.

Les méthodes de caractérisation des indicateurs retenus ont été choisies sur la base des recommandations de I'« ILCD Handbook » (JRC, 2011) :

- changement climatique : selon la méthode IPCC, 2013, l'indicateur exprimé en kg éq. CO , comptabilise les émissions de gaz à effet de serre ;

- diminution des ressources minérales et fossiles : selon la méthode CML 2002, l'indicateur exprimé en kg éq.Sb, reflète l'utilisation de ressources non renouvelables. Lindicateur prend en compte la rareté relative des différentes ressources, en considérant les réserves disponibles et le taux d'extraction ;

- consommation d'eau : exprimé en $\mathrm{m}^{3}$, l'indicateur est le volume d'eau prélevé. Pour faciliter la compréhension, un indicateur de flux a été privilégié, tandis que d'autres méthodes plus complexes existent aujourd'hui pour intégrer des facteurs de stress hydrique propres à chaque région (Kounina et al., 2013) ; - acidification des terres et des eaux douces : selon la méthode « Accumulated Exceedance », l'indicateur exprimé en kg éq. $H+$, est lié aux composés soufrés, aux composés azotés et au dioxyde de carbone qui contribuent à l'acidification de l'eau ou du sol affectant ainsi la faune et la flore ;

- eutrophisation marine: selon la méthode EUTREND, l'indicateur exprimé en kg éq. N, permet d'évaluer l'effet de l'introduction de nutriments dans les milieux aquatiques. Un trop fort apport en nutriments peut conduire à la prolifération d'algues et de planctons dans un milieu aquatique au-delà de sa capacité d'autoépuration, entraînant sa modification et sa dégradation. Deux indicateurs peuvent rendre compte de cet impact : I'eutrophisation des eaux marines (en kg équivalent azote) et l'eutrophisation des eaux douces (en kg équivalent phosphore). L'indicateur concernant l'eutrophisation marine est plus sensible à l'impact des pratiques agricoles et correspond à l'indicateur d'eutrophisation retenu dans le cadre des travaux de la plateforme ADEME/AFNOR sur l'affichage environnemental pour les produits alimentaires (AFNOR, 2012).

production, la période de référence considérée étant l'année civile 2014. La production correspondante est ainsi de 7633 tonnes de produits commercialisés, réparties en 2645 tonnes de foie gras cru et 4988 tonnes de foie gras transformé, représentant $50 \%$ de la production nationale de foie gras (PALSO, 2014). La production de foie gras transformé se répartit selon les familles de produits suivantes : $50 \%$ de foies gras entiers ; $34 \%$ de blocs avec morceaux ; $13 \%$ de blocs sans morceaux ; $3 \%$ de préparations contenant $100 \%$ de foie gras.

Les impacts environnementaux potentiels de la filière sont étudiés au travers de cinq indicateurs : $i)$ changement climatique, ii) diminution des ressources minérales et fossiles, iii) consommation d'eau, iv) acidification des terres et des eaux douces et v) eutrophisation marine (encadré 2). Le logiciel SimaPro (PréConsultant version 8.2.0.0) a été utilisé pour la modélisation ainsi que la base de données Ecoinvent v3.1. L'étude a fait l'objet d'une revue critique externe selon les recommandations des normes ISO 14040 (ISO, 2006a) et ISO 14044 (ISO, 2006b).

\subsection{Construction}

\section{d'un Inventaire de Cycle} de Vie à l'échelle de la filière

Établir l'Inventaire de Cycle de Vie (ICV) consiste à quantifier les flux entrants et sortants à chaque étape du cycle de vie pour le système étudié. Sur la base des frontières choisies, l'exercice consiste donc à comptabiliser les flux de consommations de ressources et d'émissions de polluants intervenant au cours des différentes étapes de production et de distribution ou générés par les activités des acteurs de l'environnement proche.

L'ICV de la filière est construit en plusieurs étapes : i) caractérisation de chacune des étapes du cycle de vie (élevage et gavage, abattage, transformation...), ii) inventaire des flux entrants et sortants à l'issue de chaque étape à l'échelle de la filière (nombre de canards élevés et abattus sur l'année, tonnage de produits commercialisés...) et iii) agrégation à l'échelle de la filière (Besnier et al., 2016).

La collecte de données diffère selon l'étape de cycle de vie concernée, comme présenté dans le tableau 1. En ce qui concerne les étapes amont 
Tableau 1. Collecte de données pour construire l'Inventaire de Cycle de Vie des différentes étapes étudiées et flux utilisés pour l'expression des résultats (année de référence : 2014).

\begin{tabular}{|c|c|c|}
\hline Étape & Approche utilisée & Flux de référence \\
\hline Élevage/gavage & $\begin{array}{l}\text { Inventaire type établi en collaboration avec l'INRA } \\
\text { sur la base du cahier des charges IGP (composition } \\
\text { de la ration alimentaire, émissions gazeuses...) } \\
\text { (Deneufbourg et al., } 2017 \text { et tableaux } 2 \text { et } 3 \text { ). }\end{array}$ & $\begin{array}{l}\text { Nombre de canards gavés sur l'année } \\
\text { de référence. }\end{array}$ \\
\hline Abattage/découpe & $\begin{array}{l}\text { Collecte de données annuelles d'activité auprès } \\
\text { d'un échantillon de } 7 \text { abattoirs, représentant } 37 \% \\
\text { du nombre total de canards abattus pour la filière IGP. }\end{array}$ & $\begin{array}{l}\text { Nombre de canards abattus sur l'année } \\
\text { de référence. }\end{array}$ \\
\hline Transformation & $\begin{array}{l}\text { Collecte de données annuelles d'activité auprès d'un } \\
\text { échantillon de } 7 \text { ateliers de transformation, représentant } \\
43 \% \text { du tonnage de produits transformés sous IGP. }\end{array}$ & $\begin{array}{l}\text { Poids de produits finis transformés } \\
\text { sur l'année de référence. }\end{array}$ \\
\hline Conditionnement & $\begin{array}{l}\text { Inventaire établi à partir d'une répartition globale par type } \\
\text { d'emballages selon le type de produit fini (foie gras cru, } \\
\text { foie gras transformé mi-cuit ou en conserve). }\end{array}$ & $\begin{array}{l}\text { Poids d'emballages sur l'année } \\
\text { de référence. }\end{array}$ \\
\hline Distribution & $\begin{array}{l}\text { Inventaire établi à partir de la répartition entre } \\
\text { les différents circuits de distribution et les destinations } \\
\text { géographiques finales. }\end{array}$ & $\begin{array}{l}\text { Poids de produits commercialisés } \\
\text { sur l'année de référence. }\end{array}$ \\
\hline Consommation & $\begin{array}{l}\text { Inventaire établi à partir de scénarios de conservation } \\
\text { et de préparation selon le type de produit. }\end{array}$ & $\begin{array}{l}\text { Poids de produits consommés } \\
\text { sur l'année de référence. }\end{array}$ \\
\hline Environnement proche & $\begin{array}{l}\text { Estimations des jours de bureaux et des déplacements } \\
\text { professionnels. }\end{array}$ & $\begin{array}{l}\text { Nombre d'ETP (Équivalent Temps Plein) } \\
\text { sur l'année de référence. }\end{array}$ \\
\hline
\end{tabular}

d'élevage et de gavage, les données utilisées proviennent du travail effectué par Deneufbourg et al. (2017) qui a permis à partir de sources bibliographiques et de contacts avec des experts d'établir un ICV type basé sur le cahier des charges de I'IGP. Les principales données zootechniques et hypothèses retenues sont indiquées dans le tableau 2. Le tableau 3 précise la composition de la ration alimentaire. La base de données Agribalyse v1.3 (Koch et Salou, 2014) a été utilisée pour disposer des ICV de production de chaque type d'aliment.

Un effort particulier a porté sur la collecte de données auprès des acteurs de l'abattage et de la découpe comme de la transformation, étapes pour lesquelles très peu de données sont disponibles. À partir des données annuelles de consommations d'eau, d'énergie, de consommables et de production de déchets et d'effluents fournies par l'échantillon d'entreprises interrogées ( 7 abattoirs et 7 unités de transformation), des ratios moyens ont pu être établis tels que présentés dans le tableau 4 pour l'étape d'abattage et de découpe. Pour la prise en compte du conditionnement des produits finis, des hypothèses ont été choisies pour associer à chaque famille de produit une répartition par type de conditionnement (boîte métal, bocal verre, plastique sous vide, barquette plastique) sur la base de données globales sur la répartition entre conserve et mi-cuit des produits mis sur le marché (statistiques CIFOG, 2014) et sur des informations fournies par les transformateurs ayant été enquêtés.

Pour pouvoir ensuite agréger I'ICV global au niveau de la filière, il est nécessaire de connaître les flux de produits entrants et sortants à l'échelle de la filière pour chaque étape du cycle de vie : nombre de canards abattus sur l'année de référence, tonnage total de produits finis commercialisés et destination finale, etc. Cette cartographie des flux est synthétisée sur la figure 2 . Elle a été réalisée en s'appuyant notamment sur les statistiques établies pour l'année de référence (2014) par l'organisme de défense et de gestion (PALSO) et l'interprofession (CIFOG) et sur les statistiques douanières.

Par ailleurs, I'analyse détaillée des statistiques de la filière IGP a mis en avant la problématique du déclassement technique ou commercial (non-conformité par rapport à certains critères du cahier des charges, orientation vers le marché export...) qui survient à l'issue des étapes de gavage et d'abattage/ découpe (figure 2). En effet, dans le cadre du circuit de production IGP pour l'année 2014, environ 24600000 canards sont sortis des salles de gavage selon les statistiques du PALSO tandis que sur la base du tonnage finalement commercialisé sous IGP (7 633 tonnes), le nombre théorique de canards gavés nécessaires serait d'environ 14100000 . D'un point de vue méthodologique, le choix a néanmoins été fait d'imputer à la filière IGP l'ensemble des canards mobilisés en amont du déclassement puisqu'ils font partie du circuit IGP jusqu'à leur déclassement. Dans le cadre de l'approche filière choisie dans le projet, cette méthode semble la plus cohérente car elle reflète la réalité de 
Tableau 2. Principales données de l'inventaire de cycle de vie établi pour l'élevage/ gavage (Deneufbourg et al., 2017).

\begin{tabular}{|c|c|c|c|c|}
\hline Élevage/gavage & Unité & Démarrage & $\begin{array}{l}\text { Croissancel } \\
\text { finition }\end{array}$ & Gavage \\
\hline \multicolumn{5}{|l|}{ Alimentation } \\
\hline Durée distribution & jours/canard & 28 & 55 & 10,5 \\
\hline Consommation & $\mathrm{kg} / \mathrm{canard}$ & 3,4 & 12,7 & 9,3 \\
\hline \multicolumn{5}{|l|}{ Données zootechniques } \\
\hline Durée des phases d'élevage & jours/canard & \multicolumn{2}{|c|}{83} & 10,5 \\
\hline Taux mortalité & $\%$ & \multicolumn{2}{|c|}{2,5} & 2,5 \\
\hline Poids vif (entrée) & $\mathrm{kg} / \mathrm{canard}$ & 0,044 & & 4,10 \\
\hline Poids vif (sortie) & $\mathrm{kg} / \mathrm{canard}$ & & 4,10 & 5,70 \\
\hline \multicolumn{5}{|l|}{ Litière } \\
\hline Nature & - & \multicolumn{2}{|c|}{ Paille } & - \\
\hline Quantité & $\mathrm{kg} / \mathrm{canard}$ & \multicolumn{2}{|c|}{8} & - \\
\hline \multicolumn{5}{|l|}{ Énergie } \\
\hline Consommation électricité & kWh/canard & \multicolumn{2}{|c|}{0,39} & 3,57 \\
\hline Consommation gaz & kWh/canard & \multicolumn{2}{|c|}{1,96} & 0 \\
\hline \multicolumn{5}{|l|}{ Eau } \\
\hline Consommation eau boisson & I/canard & \multicolumn{2}{|c|}{44} & 33 \\
\hline $\begin{array}{l}\text { Consommation eau pâtée } \\
\text { gavage }\end{array}$ & I/canard & \multicolumn{2}{|c|}{0} & 7,9 \\
\hline $\begin{array}{l}\text { Consommation eau bâtiment } \\
\text { (dont nettoyage) }\end{array}$ & l/canard & \multicolumn{2}{|c|}{7} & 27 \\
\hline \multicolumn{5}{|l|}{ Transport } \\
\hline $\begin{array}{l}\text { Distance depuis étape } \\
\text { amont }\end{array}$ & $\mathrm{km}$ & \multicolumn{2}{|c|}{200} & 80 \\
\hline \multicolumn{5}{|c|}{ Émissions gazeuses liées aux déjections ${ }^{1}$} \\
\hline $\begin{array}{l}\text { Émissions protoxyde d'azote } \\
\left(\mathrm{N}_{2} \mathrm{O}\right)\end{array}$ & $\mathrm{kg} /$ canard & \multicolumn{2}{|c|}{0,0046} & 0,0002 \\
\hline Émissions ammoniac $\left(\mathrm{NH}_{3}\right)$ & $\mathrm{kg} / \mathrm{canard}$ & \multicolumn{2}{|c|}{0,0800} & 0,0319 \\
\hline Émissions méthane $\left(\mathrm{CH}_{4}\right)$ & $\mathrm{kg} / \mathrm{canard}$ & \multicolumn{2}{|c|}{0,0115} & 0,0247 \\
\hline
\end{tabular}

${ }^{1}$ Calculées d'après EMEP/CEIP (2014), CORPEN (2013) et (IPCC, 2006a ; IPCC, 2006b). la filière à chaque étape. Le devenir des canards suite à leur déclassement n'est pas pris en compte (en dehors du périmètre de la filière IGP foie gras).

Par ailleurs, les étapes d'élevage/ gavage et d'abattage/découpe aboutissent à la production de foie gras mais également d'autres produits et coproduits, commercialisés en tant que viande (magrets, aiguillettes, gésiers...) ou bien valorisables dans d'autres filières (plumes, sang, viscères...). Une allocation économique $a$ été appliquée pour déterminer la part des impacts de ces étapes qu'il revient d'attribuer à la filière foie gras, basée sur des données collectées auprès d'un échantillon d'entreprises concernant le prix de vente des différents produits de découpe. Il en résulte que $55 \%$ des impacts des étapes d'élevage/gavage et abattage/découpe sont attribués à la filière foie gras (sur la base de $24 €$ valorisés par canard en sortie découpe dont $13 €$ pour le foie gras).

Les activités des acteurs de «l'environnement proche » sont comptabilisées au travers d'une estimation des jours de bureau et des déplacements professionnels affectés à la filière pour chaque catégorie d'acteurs. Les impacts environnementaux calculés reflètent ainsi l'utilisation de matériel informatique, la consommation d'utilités (énergie, eau pour les bureaux) et l'utilisation de moyens de transport (voiture, train, avion).

\subsection{Impacts environnementaux potentiels de la filière IGP Foie Gras du Sud-Ouest}

Les résultats de l'évaluation environnementale montrent que, dans le cadre du modèle établi de la filière, I'activité globale de la filière IGP Foie Gras du Sud-Ouest a entraîné pour l'année 2014 un potentiel de réchauffement climatique de 197120 tonnes équivalent $\mathrm{CO}_{2}$ et une consommation potentielle d'eau de près de 26 millions $\mathrm{m}^{3}$ (tableau 5 ). En l'absence d'études similaires en termes de périmètre et de méthodologie portant sur d'autres filières, il est difficile de mettre ces chiffres globaux en perspective. En se limitant aux étapes agricoles 
Tableau 3. Composition des rations alimentaires en \% du poids brut (Deneufbourg et al., 2017).

\begin{tabular}{|l|c|c|c|}
\hline & Démarrage & Croissance/finition & Gavage \\
\hline Blé dur & 2,5 & & \\
\hline Blé grain & 40 & 35 & \\
\hline Blé son & 3,3 & 4,5 & \\
\hline Colza tourteaux déshuilé & 7 & 4 & \\
\hline Maïs drêches & 5,5 & 7 & \\
\hline Maïs farine & & & 97 \\
\hline Maïs grain & 16 & 20,5 & \\
\hline Maïs support & 3 & 3 & \\
\hline Orge grain & 5 & 10 & \\
\hline Soja tourteaux & 6 & & \\
\hline Tournesol tourteaux & 8 & 13 & \\
\hline Carbonate calcium & 1,8 & 1,8 & \\
\hline Phosphate bicalcique & 0,5 & 0,2 & \\
\hline Sel & 0,3 & 0,2 & \\
\hline Lysine & 0,5 & 0,4 & \\
\hline Méthionine & 0,2 & 0,1 & \\
\hline Premix & & 0,3 & \\
\hline
\end{tabular}

d'élevage et de gavage, le bilan s'élève à 164910 tonnes équivalent $\mathrm{CO}_{2}$. Ce chiffre peut être mis au regard de l'estimation des émissions de gaz à effet de serre de la filière française du poulet de chair pour cette même année 2014. Les statistiques Agreste indiquent que 837200000 poulets d'un poids moyen de $1,357 \mathrm{~kg}$ ont été comptabilisés en sortie élevage (AGRESTE, 2014). Ainsi, sur la base de $2,88 \mathrm{~kg}$ éq. $\mathrm{CO}_{2}$ par $\mathrm{kg}$ de poulet (Koch et Salou, 2014), la contribution de la filière poulet de chair s'élèverait à 3271910 tonnes équivalent $\mathrm{CO}_{2}$. Même si ce type de comparaison comporte de nombreuses limites, elle indique que l'élevage/gavage en filière IGP représenterait de l'ordre de $5 \%$ des émissions de GES de la filière poulet de chair tandis que le nombre de canards gras IGP représente $3 \%$ du nombre de poulets.

Les étapes agricoles de la production de foie gras, c'est-à-dire les phases d'élevage et de gavage, (figure 3) sont les plus contributrices aux impacts environnementaux potentiels de la filière, sur l'ensemble des indicateurs étudiés. En effet, ces deux étapes contribuent à $84 \%$ des impacts potentiels sur l'indicateur de changement climatique et jusqu'à $98 \%$ pour les indicateurs d'acidification et d'eutrophisation marine potentielles. Ce résultat est cohérent avec les enseignements apportés par des études existantes. Ainsi, des $A C V$ réalisées sur un échantillon de produits carnés du berceau à l'entrée du supermarché ont mis en évi- dence que l'impact est à plus de $80 \%$ dû aux matières premières c'est-à-dire la production des animaux jusqu'au stade d'abattage (Pernollet et al., 2014).

L'alimentation des canards est à l'origine selon les indicateurs de $37 \%$ (acidification) à $91 \%$ (eutrophisation marine) des impacts potentiels pour l'élevage et $51 \%$ (acidification) à $90 \%$ (eutrophisation marine) des impacts potentiels pour le gavage. Au sein de la ration alimentaire (tableau 3 ), pendant l'étape d'élevage c'est le blé qui est à l'origine de la majorité des impacts, devant le maïs. En ce qui concerne l'étape du gavage, le maïs est à l'origine des impacts potentiels associés à l'alimentation puisque la pâtée de gavage est constituée d'un mélange maïs/eau. La consommation d'eau nécessaire à la culture du maïs (culture irriguée) est responsable de $64 \%$ de la consommation d'eau globale de l'étape de gavage et de $48 \%$ de celle de l'étape d'élevage. Les émissions gazeuses liées aux déjections des canards sont également un contributeur majeur, en particulier pour les indicateurs d'acidification ( $62 \%$ de la contribution pour l'étape d'élevage) et de changement climatique $(20 \%$ de la contribution pour l'étape d'élevage).

Pour les étapes abattage/découpe et transformation, la consommation d'énergie (électricité et gaz) est le principal contributeur pour les indicateurs considérés. Elle représente ainsi $49 \%$ de la contribution au potentiel de changement climatique pour l'abattage/découpe et $35 \%$ pour la transformation. Pour la consommation d'eau, l'eau intervenant indirectement dans la production d'électricité est à l'origine de plus de $70 \%$ de la contribution à l'indicateur ( $71 \%$ pour l'abattage/découpe ; $76 \%$ pour la transformation) tandis que l'eau directement utilisée dans le processus représente moins de $20 \%$ de la consommation globale d'eau pour les deux étapes.

En cumulant les étapes d'élevage, gavage et abattage/découpe, l'ACV réalisée montre que la production de $1 \mathrm{~kg}$ de foie gras cru entraîne un changement climatique potentiel de $13,7 \mathrm{~kg}$ éq. $\mathrm{CO}_{2}$ (tableau 6). La production de foie gras de canard semble ainsi générer des 
impacts environnementaux potentiels plus faibles que la production de foie gras d'oie qui ont été estimés à $37,5 \mathrm{~kg}$ éq. $\mathrm{CO}_{2}$ par $\mathrm{kg}$ de foie gras (Arroyo et al., 2013). Les facteurs explicatifs résident dans la meilleure valorisation économique de la viande de canard grâce aux magrets et une durée d'élevage plus courte (13 semaines contre 16 semaines pour les oies). Les résultats obtenus peuvent également être exprimés en kg de poids vif en vue de s'affranchir de la problématique d'allocation et de permettre la comparaison avec des études réalisées sur d'autres filières d'élevage. Les étapes cumulées d'élevage et de gavage génèrent $12,2 \mathrm{~kg}$ éq. $\mathrm{CO}_{2}$ pour un canard, soit $2,14 \mathrm{~kg}$ éq. $\mathrm{CO}_{2}$ par kg de poids vif. Les impacts environnementaux de la production de canard apparaissent ainsi proches de ceux décrits dans AGRIBALYSE pour la production de poulet $\left(2,88 \mathrm{~kg}\right.$ éq. $\mathrm{CO}_{2} /$ $\mathrm{kg}$ ) et de porc (2,56 éq. $\mathrm{CO}_{2} / \mathrm{kg}$ ) (Koch et Salou, 2014). Par ailleurs, le projet ANR AGRALID a produit des résultats exprimés par kg de viande mangeable. En considérant 2,575 kg de viande pour un canard gavé, le résultat obtenu est de $4,74 \mathrm{~kg}$ éq. $\mathrm{CO}_{2}$ en sortie gavage, tandis que les impacts calculés par Pernollet et al. (2014) du berceau à l'entrée du supermarché s'élèvent à : $3,79 \mathrm{~kg}$ éq. $\mathrm{CO}_{2}$ pour le poulet entier et $5,34 \mathrm{~kg}$ éq. $\mathrm{CO}_{2}$ pour le rôti de porc. La littérature scientifique fournit également des éléments de comparaison pour la consommation d'eau. Sur la base des résultats des phases d'élevage et de gavage uniquement, l'ACV conduit à une consommation potentielle d'eau de 1690 litres pour $1 \mathrm{~kg}$ de foie gras cru tandis que Corson et Doreau (2013) donnent des fourchettes d'estimations allant de 4856 à 6000 litres par kg de porc ou de 3500 à 4325 litres par $\mathrm{kg}$ de poulet en ne considérant que la phase d'élevage. II est néanmoins essentiel de noter que les valeurs sont très variables d'une étude à l'autre selon les approches choisies pour comptabiliser l'eau consommée.

L'approche filière mise en œuvre permet également un éclairage sur l'importance relative des impacts potentiels des étapes aval. Les résultats mettent en avant que l'étape de conditionnement contribue essentiellement à l'indicateur de diminution des ressources minérales
Tableau 4. Principales données de l'Inventaire de Cycle de Vie établi pour l'étape d'abattage/découpe.

\begin{tabular}{|c|c|}
\hline \multicolumn{2}{|c|}{ Bilan abattage/découpe (par canard abattu) } \\
\hline \multicolumn{2}{|l|}{ Énergie/eau } \\
\hline Électricité (kWh) & 1,52 \\
\hline Gaz (kWh) & 1,16 \\
\hline Eau (réseau) (I) & 25 \\
\hline \multicolumn{2}{|l|}{ Consommables (en g) } \\
\hline Cire pour plumaison & 30 \\
\hline Film plastique $\mathrm{PE}$ & 50 \\
\hline Caisses plastiques réutilisables & 1 \\
\hline Produits de nettoyage & 15 \\
\hline Fluides frigorigènes & 0,045 \\
\hline \multicolumn{2}{|l|}{ Déchets industriels (en g) } \\
\hline Quantité & 100 \\
\hline \multicolumn{2}{|l|}{ Effluents (I) } \\
\hline Volume effluents & 25 \\
\hline \multicolumn{2}{|l|}{ Transport amont } \\
\hline Distance (km) & 75 \\
\hline Poids canard gavé $(\mathrm{kg})$ & 5,7 \\
\hline
\end{tabular}

Figure 2. Synthèse des flux de produits intervenant aux différentes étapes.

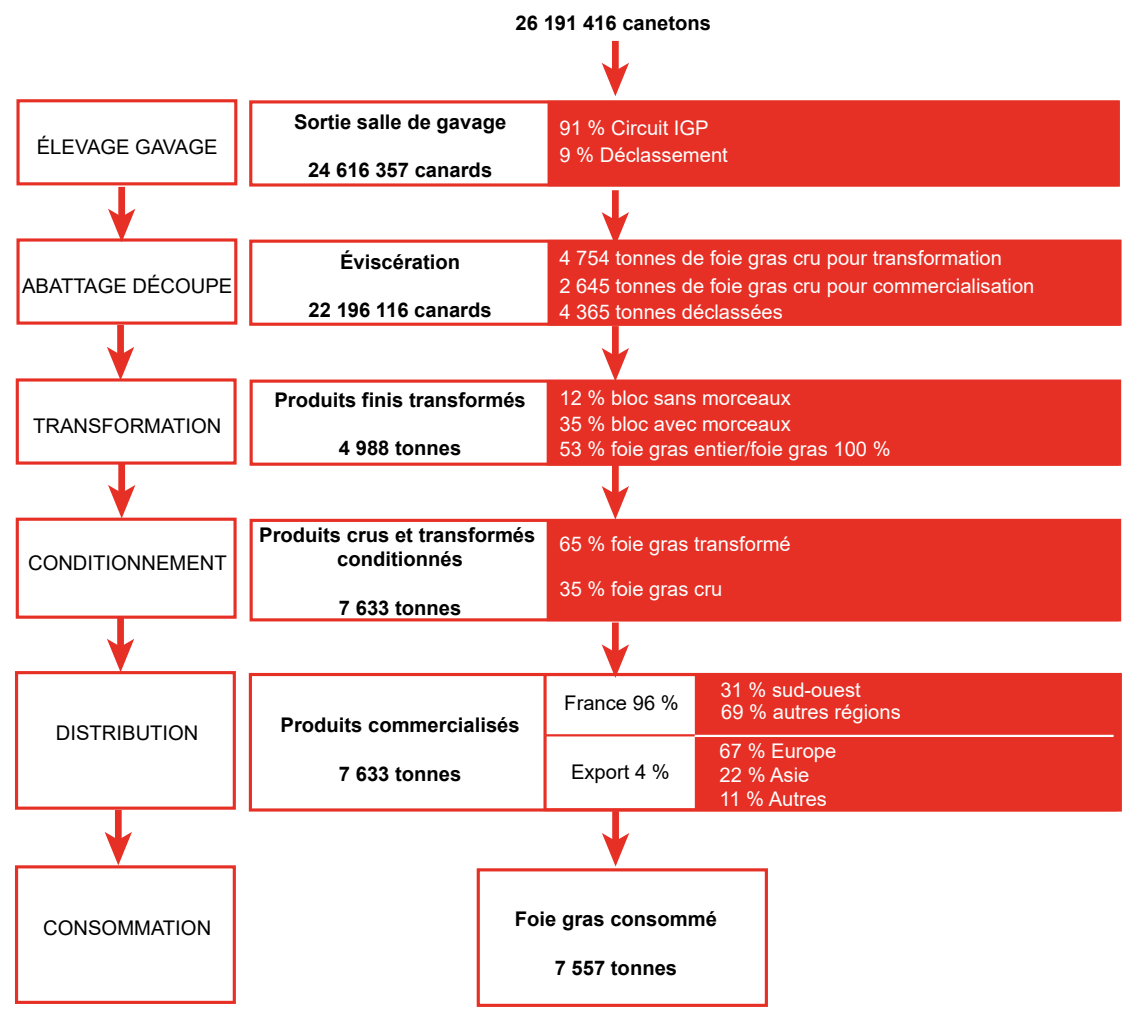


Tableau 5. Impacts environnementaux potentiels de la filière IGP foie gras du Sud-Ouest pour l'année 2014.

\begin{tabular}{|l|r|r|r|r|r|r|r|r|}
\hline \multicolumn{1}{|c|}{ Catégorie d'impact } & \multicolumn{1}{|c|}{ TOTAL } & \multicolumn{1}{c}{$\begin{array}{l}\text { Élevage/ } \\
\text { gavage }\end{array}$} & $\begin{array}{l}\text { Abattage/ } \\
\text { découpe }\end{array}$ & $\begin{array}{r}\text { Transfor- } \\
\text { mation }\end{array}$ & $\begin{array}{l}\text { Condition- } \\
\text { nement }\end{array}$ & $\begin{array}{c}\text { Distri- } \\
\text { bution }\end{array}$ & $\begin{array}{c}\text { Consom- } \\
\text { mation }\end{array}$ & $\begin{array}{c}\text { Environ- } \\
\text { nement } \\
\text { proche }\end{array}$ \\
\hline $\begin{array}{l}\text { Changement climatique } \\
\text { (kg éq. CO2) }\end{array}$ & 197118344 & 164909380 & 12187209 & 7296702 & 5969918 & 1501632 & 5093206 & 160297 \\
\hline $\begin{array}{l}\text { Acidification terrestre } \\
\text { et des eaux douces } \\
\text { (mol éq. H+) }\end{array}$ & 8178970 & 8044146 & 41962 & 14530 & 42753 & 6899 & 28004 & 676 \\
\hline $\begin{array}{l}\text { Eutrophisation marine } \\
\text { (kg éq. N) }\end{array}$ & 1647422 & 1614973 & 13078 & 3997 & 7741 & 2263 & 5232 & 139 \\
\hline $\begin{array}{l}\text { Diminution des } \\
\text { ressources minérales } \\
\text { et fossiles (kg éq. Sb) }\end{array}$ & 11794 & 9898 & 373 & 244 & 541 & 46 & 657 & 35 \\
\hline $\begin{array}{l}\text { Consommation d'eau } \\
\text { (m³) }\end{array}$ & 25964803 & 22028860 & 1857668 & 726738 & 308767 & 102083 & 935343 & 5345 \\
\hline
\end{tabular}

Figure 3. Contribution (en \%) des différentes étapes du cycle de vie aux impacts environnementaux potentiels de la filière IGP Foie Gras du Sud-Ouest.

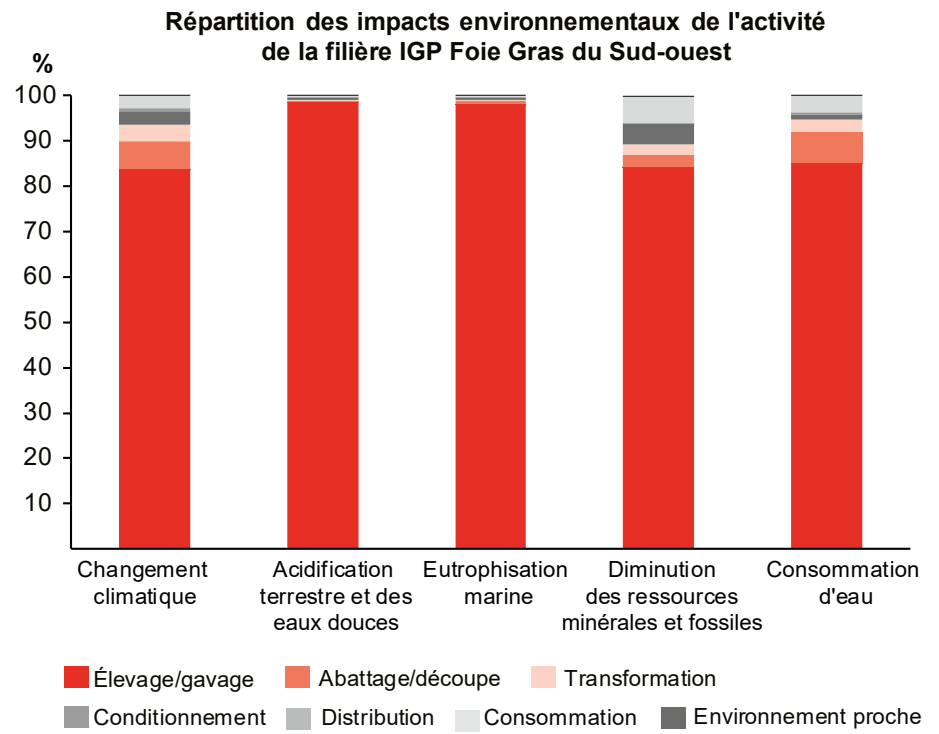

et fossiles $(4,6 \%)$ du fait des matériaux d'emballages utilisés, et notamment du verre qui intervient pour $20 \%$ du tonnage de produits commercialisés. Les bénéfices liés au recyclage du verre sont comptabilisés au niveau de l'étape de consommation durant laquelle les emballages des produits sont jetés. Un taux de recyclage de $85 \%$ est considéré (ADEME, 2013).

Les étapes distribution et environnement proche contribuent chacune à moins de 1,5\% des impacts potentiels globaux, et ce pour l'ensemble des indicateurs étudiés.

\section{2. Évaluation des performances sociales, économiques et territoriales de la filière}

Plusieurs cadres théoriques ont été développés pour définir la durabilité sociale et sa mise en application. Les approches et méthodes existantes se réfèrent à trois écoles (encadré 3 ). Celle des « attributs » mesure la performance sociale (Dreyer et al., 2006 ; Norris, 2006 ; Andrews et al., 2009 ; Kruse et al., 2009). Celle des « pathways » mesure les impacts sociaux d'une décision grâce à des relations causales et fonctionnelles (Hunkeler, 2006 ;Weidema, 2006 ; Parent et al., 2010 ; Macombe et al., 2013). Celle dite des «capacités » se concentre sur les effets d'une décision (les capacités d'Amartya Sen) de l'entreprise sur la capacité opérationnelle des acteurs aux niveaux économique, naturel, humain, social et institutionnel (Garrabé et Pedemay, 2012). Généralement les mesures sont faites au niveau d'une seule entreprise, ou pour une unité fonctionnelle d'un bien ou d'un service le long d'une chaîne de production et de distribution ; par exemple les indicateurs sont mesurés pour une production de $1 \mathrm{~kg}$ de produit emballé sortie d'usine ou pour un chiffre d'affaires de $10000 €$ ou encore pour 100 heures de travail dans la chaîne de production et de distribution. Aucune des trois approches ne permet de mesurer la performance économique et sociale d'une filière dans son ensemble sur un territoire donné. Compte tenu des limites des avancées actuelles en termes de méthode et des intérêts des professionnels, nous avons mis au point dans le cadre du projet ACYDU une nouvelle méthode hybride inspirée essentiellement de la méthode des attributs et de celle des capacités.

Les préoccupations suivantes ont jalonné notre réflexion :

- la volonté de disposer de références pour pouvoir juger/positionner la valeur mesurée des indicateurs en 
Tableau 6. Impacts environnementaux potentiels pour produire $1 \mathrm{~kg}$ de foie gras cru depuis l'étape d'élevage jusqu'en sortie abattoir en allouant $55 \%$ des impacts des étapes d'élevage, de gavage et d'abattage au foie gras.

\begin{tabular}{|l|c|}
\hline \multicolumn{1}{|c|}{ Catégorie d'impact } & $\begin{array}{c}\text { Impacts potentiels } \\
\text { pour 1 kg de foie } \\
\text { gras cru }\end{array}$ \\
\hline Changement climatique (kg éq. CO2) & 13,69 \\
\hline Acidification terrestre et des eaux douces (mol éq. $\mathrm{H}+)$ & 0,62 \\
\hline Eutrophisation marine (kg éq. $\mathrm{N})$ & 0,12 \\
\hline Diminution des ressources minérales et fossiles (kg éq. Sb) & $7,9 \times 10^{-4}$ \\
\hline Consommation d'eau ( $\left.\mathrm{m}^{3}\right)$ & 1,85 \\
\hline
\end{tabular}

termes de performance économique et sociale;

- le parti pris de mesurer des pratiques et des performances, en gardant à l'esprit que ce ne sont pas des impacts au sens propre du terme (ne disposant pas à l'heure actuelle de modèles d'impact permettant de passer des pratiques à l'échelle de l'entreprise à un impact global) mais plutôt des effets ;

- le souci de se distinguer de la position d'auditeur: I'objectif n'est pas de vérifier le respect de la réglementation ou d'un cahier des charges (les obligations légales ne serviront que de références parmi d'autres pour établir les échelles de qualification des pratiques) ;
- la volonté d'aller au-delà du simple affichage, de la vitrine, du déclaratif ;

- le positionnement à l'échelle de la filière et non à l'échelle de l'entreprise ;

- le souhait d'obtenir des résultats complémentaires aux résultats de I'ACV environnementale pour aboutir à l'outil de mesure de la contribution de la filière au développement durable du territoire ;

- la volonté d'une méthode suffisamment générique pour s'adapter à d'autres filières.

Cette méthode vise à fournir un outil opérationnel qui peut être combiné avec l'analyse du cycle de vie environnementale pour décrire les pratiques sociales, mesurer les performances socio-économiques d'une filière et des opérateurs la composant sur le territoire. Le même périmètre que celui de l'ACV environnementale a été appliqué (figure 1). De même l'unité fonctionnelle retenue est la quantité de produits finis commercialisés par la filière au cours d'une année. Afin de considérer la diversité des formes d'organisation existant dans la filière, la collecte des données a été organisée selon les différents circuits qui coexistent. Chacun est constitué d'opérateurs qui regroupent une ou plusieurs étapes de la fabrication du produit selon des caractéristiques de volume, de statut et de partenaires amont-aval.

\subsection{Méthodologie} de mesure des performances

\section{a. Principes à la base de la méthode}

L'ACV sociale ou ACV-S comporte des indicateurs sociaux mais aussi économiques. Les lignes directricesUNEP/ SETAC (2009) rapportent explicitement: "l'expression analyse sociale du cycle de vie sera utilisée comme synonyme de l'expression analyse sociale et socioéconomique du cycle de vie ». De plus, il est parfois difficile de classer certains indicateurs dans la case "économique » ou dans la case « sociale » (exemple : la rémunération des travailleurs). Ainsi il a été choisi d'appliquer la démarche

\section{Encadré 3. Les 3 approches de l'ACV-Sociale.}

L’Analyse sociale du Cycle de Vie est couramment appréhendée selon trois approches:

- Approche des attributs : I'objectif est de mesurer la performance d'une entreprise. Elle se situe dans le droit fil des travaux sur la Responsabilité Sociale des Entreprises (RSE). C'est une approche micro/méso qui révèle le point d'observation des seuls opérateurs de la filière. Elle ne permet pas de mesurer véritablement les impacts sociaux puisqu'elle se limite aux performances économiques et sociales des entreprises. Elle utilise des indicateurs intermédiaires (« mid-point ») et se limite à l'entreprise.

- Approche des «pathways »: son but est de prédire les impacts sociaux sur les populations, liés à des changements dans le fonctionnement d'une chaîne de production tout au long de son cycle de vie. Elle recherche les relations causales et fonctionnelles statistiquement significatives entre des facteurs et des impacts (relations causes-effets). II s'agit d'une approche méso/macro qui ignore les spécificités des conditions locales et ne rend pas compte de l'impact d'une filière. Elle utilise des indicateurs finaux (« end-point ») qui représentent une valeur pour la société.

- Approche des capacités : I'objectif est de mesurer dans un certain contexte local, les effets économiques, humains, sociaux, financiers et institutionnels d'une décision et d'une action des entreprises (pour chaque niveau de filière, pour chaque catégorie d'acteur). Un modèle des capitaux multiples permet de mesurer I'impact d'une action de l'entreprise sur la capacité opérationnelle des différents acteurs d'une filière. Cette approche peu intuitive ne permet pas des comparaisons entre secteurs.

Exemple : la mise à disposition d'une formation pour les salariés. Dans l'approche des attributs, c'est un indicateur de performance. Dans l'approche des «pathways», il s'agira de mesurer l'impact (santé, bien-être, gain social) de cette formation au niveau national. Pour l'approche des capacités, mettre à disposition une formation n'implique pas qu'elle soit effectivement suivie et qu'elle ait des effets sur les connaissances des salariés. Il y aura un réel effet quand le savoir sera transformé en compétence acquise. 
140 / LAURA FARRANT et al.

Tableau 7. Synthèse des catégories, sous-catégories, indicateurs.

\begin{tabular}{|c|c|c|}
\hline Catégories de performance & Sous-catégories & Indicateurs \\
\hline \multirow{21}{*}{$\begin{array}{l}\text { Dignité et bien-être } \\
\text { des travailleurs }\end{array}$} & \multirow{3}{*}{ Santé et sécurité } & $\begin{array}{l}\text { Taux de gravité des accidents du travail et maladies } \\
\text { professionnelles }\end{array}$ \\
\hline & & Pénibilité des conditions de travail \\
\hline & & Santé et sécurité des travailleurs \\
\hline & \multirow{3}{*}{ Cadre de travail } & $\begin{array}{l}\text { Organisation d'évènements et aménagements } \\
\text { exceptionnels }\end{array}$ \\
\hline & & Aménagement du temps de travail \\
\hline & & Mise à disposition d'un local social \\
\hline & \multirow{5}{*}{ Avantages aux salariés } & Avantages en nature \\
\hline & & Avantages type CE \\
\hline & & Complémentaire retraite \\
\hline & & Complémentaire santé \\
\hline & & Avantages économiques \\
\hline & Représentation des salariés & Espace de discussion Direction/salariés \\
\hline & \multirow{4}{*}{ Limiter la précarité } & Respect des horaires de travail et de repos \\
\hline & & Facilités mises à disposition des saisonniers \\
\hline & & \begin{tabular}{|l|} 
Pérennisation des saisonniers \\
\end{tabular} \\
\hline & & Limiter les saisonniers d'Etats tiers \\
\hline & \multirow{5}{*}{ Caractéristiques de l'emploi } & Qualification \\
\hline & & Rémunération ouvrier \\
\hline & & Rémunération cadre \\
\hline & & Egalité homme - femme chez les cadres \\
\hline & & Représentation des femmes \\
\hline \multirow{6}{*}{ Territoire et vie locale } & \multirow{2}{*}{ Intégration dans la vie locale } & Participation aux évènements locaux \\
\hline & & Nuisances \\
\hline & \multirow{7}{*}{ Engagements de l'entreprise } & Promotion de l'identité du territoire \\
\hline & & Préservation du patrimoine \\
\hline & & Reconnaissance institutionnelle \\
\hline & & Image du produit \\
\hline \multirow{11}{*}{$\begin{array}{l}\text { Loyauté, intégrité } \\
\text { des pratiques }\end{array}$} & & Engagement RSE \\
\hline & & Engagements en sécurité sanitaire et qualité \\
\hline & & Engagements environnementaux \\
\hline & \multirow{4}{*}{ Sous-traitance } & Motif \\
\hline & & Pénibilité \\
\hline & & Engagement environnemental, social et de santé \\
\hline & & Respect des conditions de travail \\
\hline & \multirow{4}{*}{ Relations aux partenaires } & $\begin{array}{l}\text { Pratiques sociales et environnementales des } \\
\text { concurrents }\end{array}$ \\
\hline & & Fidélité aux partenaires \\
\hline & & Organisation d'évènements avec les partenaires \\
\hline & & Implication dans un projet commun \\
\hline \multirow{13}{*}{ Création de richesse } & \multirow{11}{*}{ Richesse matérielle } & Taux de valeur ajoutée \\
\hline & & Poids masse salariale \\
\hline & & Efficacité économique \\
\hline & & Taux d'investissement \\
\hline & & Résultat net en \% du CA \\
\hline & & Taux de dépendance aux aides \\
\hline & & Répartition du résultat \\
\hline & & Capacité d'autofinancement \\
\hline & & Rentabilité \\
\hline & & Taux d'endettement \\
\hline & & Équilibre financier \\
\hline & \multirow{2}{*}{ Richesse immatérielle } & Taux d'accès à la formation \\
\hline & & Relations avec Formation et Recherche \\
\hline
\end{tabular}


du cycle de vie pour couvrir simultanément les domaines sociaux et économiques dans leurs effets à la fois au niveau des individus et de la filière dans son ensemble, mais aussi au niveau du territoire spécifique de I'IGP.

Notre démarche n'est pas à proprement parler participative, au sens de l'implication des parties prenantes dans l'exercice d'évaluation. Cependant les discussions autour de notre questionnaire avec les organismes, entreprises, personnes qui ont accepté de nous rencontrer ont nécessairement engagé ou prolongé la réflexion sur l'appartenance à une filière, les impacts et la durabilité de ses activités.

En pratique, partant d'une revue de littérature et des questions intéressant les professionnels de la filière, nous avons mêlé ces trois domaines d'intérêt (l'économique, le social et le territorial) et retenu quatre catégories pour cette méthode hybride :i) dignité et bien-être des travailleurs, ii) territoire et vie locale, iii) loyauté et intégrité des pratiques commerciales, iv) création de richesse. Ces quatre catégories sont subdivisées en 13 sous catégories (seules 12 d'entre elles seront ici illustrées car la soustraitance n'est pas utilisée dans I'IGP foie gras), elles-mêmes caractérisées par 50 indicateurs (tableau 7). Les indicateurs ont été sélectionnés pour leur pertinence et leur opérationnalité mais aussi par rapport à l'existence de l'information et sa disponibilité. Ils ont été choisis de façon à éviter les redondances et discutés avec les professionnels rencontrés lors des entretiens. Cette méthode générique est applicable à toute filière de produits agroalimentaires transformés. Elle vise à repérer où se situent les marges de progrès pour qualifier la filière comme socialement durable.

\section{b. Construction de la méthode}

La méthode proposée est en fait un modèle d'évaluation multicritères allant jusqu'à une évaluation globale de la performance socio-économique de la filière. Pour cela, l'équipe du projet ACYDU a décidé que les 50 indicateurs seraient renseignés grâce à l'attribution d'un score (noté entre 0 et 10) ; chacun d'entre eux est évalué par rapport à un niveau de référence international (par exemple, les normes internationales du travail du Bureau International du Travail); à défaut la référence sera nationale ou sectorielle (par exemple, le taux de valeur ajoutée du secteur et/ou des industries agroalimentaires). À titre d'exemple, dans le cas d'un indicateur quantitatif tel que l'efficacité économique de l'entreprise, les points ont été attribués selon les ratios obtenus (valeur ajoutée/production en \%) et la classe dans laquelle est située l'entreprise : 0 point si le ratio est <à $35 \%$; 3 points si le ratio est entre 35 et $50 \% ; 7$ points si le ratio est entre 50 et $65 \% ; 10$ points si le ratio est $>$ à $65 \%$. Pour un indicateur qualitatif tel que la mesure de la pénibilité, le gouvernement français avait défini (décret $\mathrm{n}^{\circ}$ 2014-1159 du 9 octobre 2014) 10 critères (manutentions manuelles de charges lourdes, postures pénibles, vibrations mécaniques, milieu hyperbare, agents chimiques dangereux, y compris poussières et fumées, températures extrêmes, travail de nuit, travail en équipes successives alternantes, travail répétitif, bruits). Pour un tel critère qualitatif, le score sera 0 s'il y a au moins un des critères de pénibilité et une absence de mesures de diminution de la pénibilité ; il sera de 7 en présence de critères de pénibilité mais avec des mesures de compensation/diminution; il sera de 10 s'il y a élimination de la pénibilité ou aucun des critères de pénibilité.

La méthode des scores permet l'agrégation des résultats et une visualisation claire des efforts à fournir en termes de performances par rapport à un optimum à atteindre. Les indicateurs sont équi-pondérés volontairement ; liberté est donnée à l'utilisateur de pondérer les quatre catégories d'indicateurs en fonction de ses objectifs.

En complément de ce modèle d'évaluation multicritère, et pour mieux appréhender les effets économiques et sociaux sur le territoire, nous avons adopté la «méthode des multiplicateurs »(Garrabé, 2014), consistant à appliquer un multiplicateur au revenu initial généré par l'activité.

Pour passer de chaque opérateur individuel au résultat à l'échelle de la filière, nous agissons en deux étapes : i) création $d^{\prime}$ ' opérateurs-types » en pondérant les scores individuels par la part du volume qu'ils représentent dans le volume total de l'ensemble des opérateurs du même type enquêtés ; ii) application d'un coefficient représentant l'importance relative en volume de ce type d'acteur dans la filière à chaque score de "l'opérateur-type ». Par exemple, pour le foie gras, nous avons considéré les opérateurs-types suivants : les conserveurs à la ferme, les entreprises autonomes, les entreprises intégrées. Pour chaque sous-catégorie puis pour chaque catégorie, le score en $\%$ du score maximal possible est calculé. Enfin, le score final de la filière est la somme des scores des différentes catégories, ramenée au score maximal total de la filière.

\subsection{Application à la filière Foie Gras du Sud-Ouest : résultats et discussion}

Un test d'épreuve sur la filière IGP Canard à foie gras du Sud-Ouest a été effectué à partir d'enquêtes auprès de 11 opérateurs de production et transformation appartenant au cœur de la filière (Assogba, 2015) représentant $41 \%$ du volume de la filière foie gras IGP (pour l'année 2013). L'objectif était d'avoir un échantillon représentatif de la diversité de la filière. Ainsi les opérateurs représentent diverses étapes de l'élaboration du produit (élevage, gavage, abattage, découpe, conservation, transformation) et sont de tailles très variables (du producteur à la ferme aux plus grandes entreprises internationales). Les entreprises ont été regroupées selon trois types d'organisation ; les conserveurs à la ferme, les entreprises autonomes et les entreprises intégrées qui représentent respectivement 1,14 et $85 \%$ du total du volume produit. Les conserveurs à la ferme concentrent toutes les activités au sein de l'entreprise ; ils vendent localement. Les entreprises autonomes produisent le foie gras en toute indépendance tandis que les entreprises intégrées font partie d'un groupe intégrant plusieurs acteurs maillons de la chaîne de production.

Les résultats ainsi obtenus à l'échelle de la filière montrent que la filière est globalement très performante avec 
Figure 4. Performances (en \% du maximum possible) de la filière IGP Foie Gras du Sud-Ouest par sous-catégories d'indicateurs.

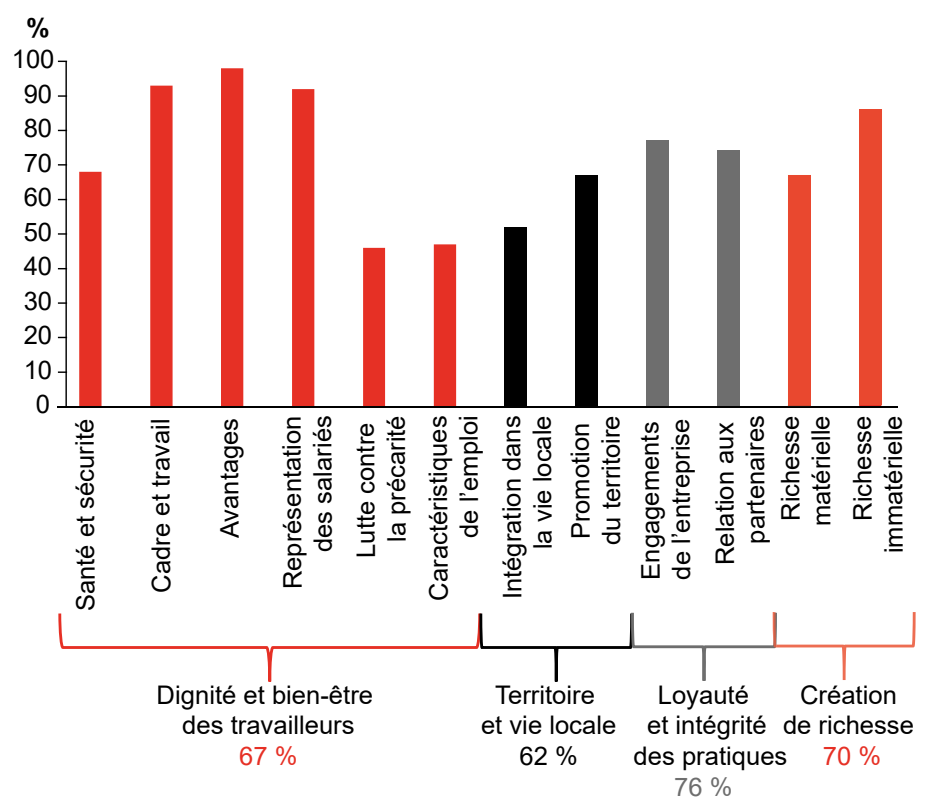

un score total de $69 \%$ par rapport au maximum possible (en considérant l'ensemble des 50 indicateurs). En analysant plus dans le détail tel que montré dans la figure 4, on observe au niveau de la composante « dignité et bien-être des travailleurs » atteignant $67 \%$, que si le cadre de travail, les avantages offerts aux salariés (en nature, avantages économiques ou de type $\mathrm{CE}$, complémentaires santé et retraite) et la capacité de représentation des salariés, sont très satisfaisants (92 à $98 \%$ ), la prise en compte de la santé et la sécurité des salariés (pénibilité, accidents du travail) a encore des marges de progrès (score à $68 \%$ ). Les caractéristiques de l'emploi et les mesures prises pour limiter la précarité sont quant à elles à reconsidérer (scores à 46-47\%).

La « loyauté et l'intégrité des pratiques commerciales » (relations aux partenaires, fidélité, projets communs, engagements des entreprises au niveau sanitaire, de la qualité, de l'environnement) est particulièrement élevée (76\%) dans la filière. Nous observons un paradoxe : alors que l'étude porte sur une filière IGP par définition très ancrée au territoire, c'est la composante « territoire et vie locale » qui est la plus faible (62\%). Ces résultats sont très influencés par ceux des entreprises intégrées au regard de leur poids relatif dans la filière $(85 \%$ du volume produit). Or si elles promeuvent largement le territoire et son image à des fins commerciales, elles participent peu à la vie locale tout comme les entreprises autonomes.

Au niveau de la création de richesse, on constate une bonne performance globale de la filière $(70 \%)$ malgré un taux de dépendance aux aides assez élevé (score de 5,3/10) et une rentabilité économique faible (4,8/10). Quant à la richesse immatérielle (formation et recherche), elle n'est pas à la portée des conserveurs à la ferme et le bon score de $86 \%$ de la filière est essentiellement dû au résultat des entreprises intégrées (94\% contre $43 \%$ pour les conserveurs à la ferme).

Pour l'étude de la richesse et l'emploi créés sur le territoire, les comptes de résultat des entreprises de l'échantillon nous ont permis d'estimer les consommations intermédiaires locales et non locales. II apparaît que $91 \%$ des consommations sont locales; les $9 \%$ restants étant surtout dus à l'achat de matériel hors zone IGP. La méthode des multiplicateurs nous permet de conclure que la filière IGP Foie Gras du Sud-Ouest est source de 46693 emplois sur la zone IGP; la production d'une tonne de foie gras crée 5,77 emplois ; le système de production transformation de la filière crée un revenu de 2,8 milliards d'euros sur la zone IGP. Cela représente environ 3,5 \% des emplois totaux et de la richesse de la région Midi-Pyrénées (INSEE, 2013 ; comptes régionaux des ménages), mais aussi $48 \%$ des emplois du secteur agro-alimentaire de la Région (INSEE, MSA).

Ces résultats sont fortement dépendants du parti pris méthodologique. Trois points peuvent être discutés :

i) le choix d'utiliser une moyenne pondérée par le volume autorise de fait un petit opérateur avec une mauvaise évaluation socio-économique à être compensé par les résultats de l'ensemble des autres opérateurs. Notre volonté de raisonner au niveau de la filière dans son ensemble, masque en effet les mauvais résultats d'une minorité. Ayant procédé pour mémoire à une évaluation des performances en pondérant par le nombre d'unités travail humain, les ordres de grandeur sont similaires. L'intérêt est ici que chaque entreprise mesure les efforts restant à fournir pour améliorer sa durabilité sociale ;

ii) les indicateurs sont équipondérés car nous nous refusons à juger de la supériorité de certains critères sur les autres. La liberté est offerte aux entreprises pour réaliser leur propre pondération entre les catégories ou sous-catégories du modèle, en fonction de leurs priorités ;

iii) le référentiel de chaque indicateur est de première importance. II doit être adapté à chaque filière et chaque pays car de lui dépend l'estimation de la performance de chaque critère.

\section{Conclusion}

L'analyse des impacts environnementaux de la filière IGP Foie Gras du Sud-Ouest a souligné la prépondérance des phases d'élevage et de gavage des canards dans les impacts globaux de la filière. Mais au-delà de ce résultat, les résultats détaillés fournissent de riches enseignements sur l'origine des impacts environnementaux pour chaque étape de la filière de production et de distribution. L'identification des principaux contributeurs aux impacts potentiels suggère des pistes d'amélioration à étudier pour réduire le bilan 
environnemental de la filière. Les leviers d'actions concernent principalement le mode d'alimentation des canards (choix et origine des matières premières composant la ration alimentaire, cf. Arroyo et al., 2013) et les pratiques d'élevage (par exemple la gestion des déjections), la conception des emballages (allègement, utilisation de matières recyclées et recyclables, dans le respect des contraintes sanitaires propres au secteur alimentaire) et l'optimisation des consommations énergétiques des ateliers d'abattage et de transformation.

Une analyse détaillée et exhaustive de l'ensemble des flux de produits et de matières depuis la « mise en place » des canetons mâles de un jour en élevage jusqu'au foie gras cru ou transformé arrivé dans l'assiette du consommateur a été réalisée pour établir l'inventaire de cycle de vie. Cette analyse propose une vision inédite de la filière IGP aux professionnels. Chaque acteur de la filière entrant dans le périmètre de notre étude peut ainsi comprendre quels flux de matières ou d'énergie entraînent des impacts potentiels à son niveau et plus largement sur le cycle de vie. Cette vision englobante à l'échelle de la filière peut rendre le travail d'écoconception plus efficace en permettant aux entreprises de cibler les actions sur les étapes les plus contributrices et/ou en stimulant les collaborations entre des acteurs dont les actions sont liées dans le cycle de vie du produit.

Pour l'analyse des performances sociales et économiques, rappelons que l'approche choisie repose sur i) la décision de mesurer les pratiques et les performances plutôt que les impacts ; ii) le désir d'utiliser des références pour juger/positionner la performance et les indicateurs mesurés; iii) I'identification des domaines à améliorer sans prise en compte de la conformité réglementaire ; iv) la volonté d'une méthode suffisamment générique pour s'adapter à d'autres secteurs. Dans ce cadre, la méthode s'avère pleinement opérationnelle. Les enseignements de ces évaluations pour la filière IGP Foie Gras du Sud-Ouest montrent que le lien au territoire au travers d'une promotion et d'un ancrage dans la vie locale est important mais le niveau de performance est hétérogène selon les entreprises ; l'enjeu lié aux emplois précaires (évalué au travers de la sous-catégorie « lutte contre la précarité ») est pris en compte en partie avec une réorganisation de la production sur toute l'année mais le recours aux saisonniers reste important et nécessaire ; l'adhésion à l'IGP et à l'interprofession facilite l'engagement des entreprises dans des actions sur les problématiques majeures de la filière.
Le travail réalisé s'est toutefois heurté aux limites de l'exercice, notamment l'énergie considérable qui doit être déployée pour mener à bien une collecte de données terrain approfondie, s'efforçant de respecter des critères de représentativité bien que la transmission de données par les entreprises relève du volontariat. Pour les données socio-économiques, certains éléments sont considérés comme confidentiels par les entreprises (questions salariales, du bien-être des travailleurs, données comptables) ; par ailleurs la question des références pour chaque critère est fondamentale car d'elle dépend toute l'estimation.

Les méthodologies proposées au travers des études de cas menées dans le cadre du projet ACYDU présentent certaines limites. Néanmoins ces travaux novateurs contribuent à faire progresser la recherche sur le couplage de différentes approches environnementales et socio-économiques pour l'analyse de la durabilité des filières agroalimentaires dans leur intégralité. Les analyses sociales et économiques ajoutent une dimension supplémentaire à l'étude d'impact environnemental : elles délivrent une information précieuse à ceux qui cherchent à produire, à acheter ou à vendre de manière responsable.

\section{Références}

ADEME, 2013. Emballages ménagers. Collection repères.

AFNOR, 2012. Principes généraux pour l'affichage environnemental des produits de grande consommation-Partie 15 : méthodologie d'évaluation des impacts environnementaux des produits alimentaires. BP X30-323-15, 0ctobre 2012, 23p.

AFNOR, 2015. Management environnemental Analyse du cycle de vie - Exigences et lignes directrices pour l'analyse du cycle de vie organisationnelle. Norme française XP ISO/TS 14072, Février 2015, indice classement $X 30-048,28 p$.

AGRESTE, 2014. Statistiques agricoles pour l'année 2014.

Andrews E., Lesage P., Benoît C., Parent J., Norris G.A., Revéret J.P., 2009. Life Cycle Attribute Assessment: Case Study of Quebec Greenhouse Tomatoes. J. Ind. Ecol., 13, 565-578.

Arroyo J., Aubin J., Auvergne A., Dubois J.P., Brachet M., Fernandez X., Debaeke P., Fortun-Lamothe L.,
2013. Conception et évaluation d'un système innovant de production de foie gras : cas de la substitution du mais par du sorgho chez l'oie. In : Palipèdes à foie gras. Fortun-Lamothe L. (Ed). Dossier, INRA Prod. Anim., 26, 435-448.

Assogba G., 2015. Évaluation des dimensions, sociales, environnementales et territoriales de filières agroalimentaires sous signes officiels de qualités. Discussion des méthodes et des premiers résultats du projet ACYDU appliqué aux filières IGP Canards Gras du Sud-Ouest, AOP Comté et AOP Vins de Bourgogne. $52^{\mathrm{e} C o l l o q u e ~ A S R D L F . ~}$

Besnier A., Farrant L., Penavayre S., Bosque F., Labau M.P., LempereurV., Pernet C., Berner J.L, Jolibert F., 2016. LCl of food products sectors: from field data to the big picture. $10^{\text {th }}$ Int. Conf. Life Cycle Assess. Food, Dublin, Irlande.

\section{CIF0G, 2014. Rapport économique annuel.}

Commission Européenne 2013 RECOMMANDATION DE LA COMMISSION du 9 avril 2013 relative à I'utilisation de méthodes communes pour mesurer et indiquer la performance environnementale des produits et des organisations sur l'ensemble du cycle de vie (2013/179/UE)

CORPEN, 2013. Normes CORPEN Volailles. Ed CORPEN, Paris, France.

Corson M.S., Doreau M., 2013. Évaluation de I'utilisation de l'eau en élevage. INRA Prod. Anim., 26, 239-248.

Deneufbourg C., Arroyo J., Wilfart A., Farrant L., Litt, J. Fortun-Lamothe L., 2017. Impacts environnementaux de 3 systèmes de production contrastés de foie gras de canard. Journ. Rech. Avicole et Palmipèdes à Foie Gras. Tours, France, 12, 897-901.

Dreyer L.C., Hauschild M., Schierbeck J., 2006. A Framework for Social Life Cycle Impact Assessment. Int. J. Life Cycle Assess., 11, 88-97.

EMEP/CEIP, 2014. Present state of emission data.

FA0, 2016. Food and Agriculture Organization of the United Nations - LEAP Guidelines for assessment. 
Garrabé M., Pedemay A., 2012. MCM-ASCV des Capacités. Application à la filière tomate industrielle en France. Rapport 5 du projet ANR Alia FLONUDEP.

Garrabé M., 2014. Valeur d'activité totale (V.A.T.) d'une opération de développement. Séminaire 3 - UM1-IAMM.

Hunkeler D., 2006. Methodology and Case Study. Int. J. Life Cycle Assess., 11, 371-382.

IPCC, 2006a. Guidelines for national greenhouse gas inventories. Vol. No 1: General guidance and reporting (GGR). Eggleston S., Buendia L., Miwa K., Ngara T., Tanabe K. (Ed). IGES, Kanagawa, Japan.

IPCC, 2006b. Guidelines for national greenhouse gas inventories. Vol. No 4: Agriculture, forestry and other land use (AFOLU).

ISO, 2006a. Management environnemental - Analyse du cycle de vie - Principes et cadre. NF EN ISO 14040, 0ctobre 2006, 23p.

ISO, 2006b. Management environnemental - Analyse du cycle de vie - Exigences et lignes directrices. EN ISO 14044, Juillet 2006, 49p.
JRC, 2011. European Commission ILCD Handbook: Recommendations for Life Cycle Impact Assessment in the European context. First edition.

Commission Européenne, 2013. Recommandation de la Commission du 9 avril 2013 relative à I'utilisation de méthodes communes pour mesurer et indiquer la performance environnementale des produits et des organisations sur l'ensemble du cycle de vie (2013/179/UE)

Koch P., Salou T., 2014. AGRIBALYSE : rapport méthodologique - version 1.1 In : ADEME (Ed). Angers, France.

Kounina A., Margni M., Bayart J.B., Boulay A.M. Berger M, Bulle C., Frischknecht R., Koehler A., Milà i Canals L., Motoshita M., Nunez M., Peters G., Pfister S., Ridoutt B., Van Zelm R., Verones F., Humbert S., 2013. Review of methods addressing freshwater use in life cycle inventory and impact assessment. Int. J. Life Cycle Assess., 18, 707-721.

Kruse S.A., Flysjö A., Kasperczyk N., Scholz A.I., 2009. Socioeconomic indicators as a complement to life cycle assessment - an application to salmon production systems Int. J. Life Cycle Assess., 14, 8-18.
Macombe C., Leskinen P., Feschet P., Antikainen R., 2013. Social life cycle assessment of biodiesel production at three levels: a literature review and development needs. J. Clean. Prod., 52, 205-216.

Norris G.A., 2006. Social Impacts in Product Life Cycles - Towards Life Cycle Attribute Assessment. Int. J. Life Cycle Assess., 11, 97-104.

PALS0, 2014. Données statistiques de la filière IGP Canards à Foie Gras du Sud-Ouest.

Parent J., Cucuzzella C., Revéret J.P., 2010. Impact assessment in SLCA: sorting the SLCIA methods according to their outcomes. Int. J. Life Cycle Assess., $15,164-171$.

Pernollet F., Thornes J., Van der Werf H.M.G, 2014. Impacts environnementaux de produits carnés et améliorations possibles. $15^{\text {es }}$ Journ. Sci. Muscle et Technologies des Viandes, Clermont Ferrand, France.

UNEP/SETAC, 2009. Guidelines for Social Life Cycle Assessment of Products. 104p.

Weidema B.P., 2006. The Integration of Economic and Social Aspects in Life Cycle Impact Assessment. Int. J. Life Cycle Assess., 11, 89-96.

\section{Résumé}

Les filières agroalimentaires font face à de multiples enjeux et questionnements en lien avec les différents aspects du développement durable. L'objectif de ces travaux est de proposer des méthodologies permettant d'aborder l'évaluation de la durabilité des filières de produits agroalimentaires transformés en s'intéressant aux impacts environnementaux ainsi qu'aux performances socio-économiques de la filière Indication Géographique Protégée (IGP) foie gras du Sud-Ouest en lien avec son implantation territoriale. Les impacts environnementaux sont étudiés via la méthode de l'Analyse de Cycle de Vie (ACV). Les résultats fournissent un éclairage approfondi sur les principaux contributeurs aux impacts de la filière pour chaque étape, depuis les phases amont d'élevage et de gavage, prépondérantes dans le bilan global de la filière, jusqu'à la consommation finale. Une nouvelle méthode est également proposée pour évaluer la performance sociale, économique et territoriale d'une filière alimentaire. La mesure des performances s'articule en quatre catégories : $i$ ) dignité et bien-être des travailleurs, ii) territoire et vie locale, iii) loyauté et intégrité des pratiques commerciales et iv) création de richesse, pour lesquelles un ensemble d'indicateurs d'évaluation est défini. Cette méthode vise à repérer où se situent les marges de progrès pour qualifier la filière en termes de performances socio-économiques. L'approche mise en œuvre souligne que la filière est globalement très performante sur les dimensions étudiées avec néanmoins des enjeux concernant la précarité de certains emplois et des voies d'amélioration concernant la participation à la vie locale.

\section{Abstract}

\section{Protected Geographical Indication "South West duck foie gras" sector: sustainability assessment}

The objective of this work is to propose methodologies in order to assess the sustainability of agri-food sectors by investigating the environmental impacts together with the social and economic performances of the Protected Geographical Indication (PGI) South West duck "foie gras" sector in connection with its territorial anchorage. Environmental impacts were assessed through Life Cycle Assessment. The results provide a detailed overview of the main contributors to the environmental impacts for each stage, from rearing and overfeeding that play a major role in the global balance, down to final consumption. A new method is also proposed to assess the social, economic and territorial performances of an agri-food sector, relying on four major categories:i) dignity and welfare of workers, ii) territory and local life, iii) loyalty and integrity of commercial practices, iv) and wealth creation. For each category a set of indicators is defined and assessed. This method aims at pointing out margins for progress to describe the sector in terms of its social and economic performances. When applied to the "foie gras" sector, this approach has shown that this sector performs well but has highlighted some concerns regarding precarious work and possible improvement concerning the involvement in local life.

FARRANT L., LABAU M.P., PADILLA M., DENEUFBOURG C., FORTUN-LAMOTHE L., PENAVAYRE S., BESNIER A., 2018. Évaluation de la durabilité de la filière Indication Géographique Protégée « Canard à foie gras du Sud-Ouest ». INRA Prod. Anim., 31, 131-144.

https://doi.org/10.20870/productions-animales.2018.31.2.2319 